\title{
Management of bleeding varices in the elderly
}

\author{
S W Hosking, N C Bird, A G Johnson, D R Triger
}

\begin{abstract}
In 207 consecutive patients the outcome of bleeding varices was compared in those aged under 65 $(n=146)$ and those aged over $65(n=61)$. All patients were seen during seven years and were treated by active initial and maintenance sclerotherapy. Alcoholic cirrhosis was commoner in younger patients $(68(47 \%) v 12(20 \%))$ and cryptogenic cirrhosis commoner in older patients $(21(34 \%) v$ $19(13 \%))$. Mortality due to the first bleed was dependent on the severity of liver disease and was unrelated to age. Survival corrected for age (life table analysis) was $65 \%$ at one year and $60 \%$ at two years for both groups of patients.

It is concluded that patients should not be denied active treatment for bleeding varices on the basis of age alone. In order to obtain optimum results early endoscopy and sclerotherapy is essential.
\end{abstract}

\section{Introduction}

Upper gastrointestinal bleeding in the elderly carries a worse prognosis than in younger patients, the prognosis being particularly poor when bleeding is due to varices.' Until recently shunt surgery or conservative treatment was the only option available; shunt surgery carries a very high risk of encephalopathy in older patients, and half of all patients treated conservatively rebleed within six months.' Injection sclerotherapy has now become accepted as an effective alternative to shunt surgery and is suitable for all ages. We report our experience of treating elderly patients with this technique and compare the results with those obtained in younger patients to ascertain whether an active sclerotherapy programme benefits the elderly.

\section{Patients and methods}

Between 1980 and 1987 we admitted 207 patients to our acute gastrointestinal unit with a first variceal bleed. Sixty one were over 65 at their initial presentation. All patients received injection sclerotherapy within 12 hours of admission, but this was preceded by balloon tamponade in those who were bleeding at their initial endoscopy. Most patients had sclerotherapy performed under sedation with a flexible endoscope, 21 having the procedure under a general anaesthetic. Patients in whom bleeding was still uncontrolled had emergency surgery.

All patients surviving their first bleed were managed by maintenance sclerotherapy, apart from 14 (all under 65) who had oesophageal transection and devascularisation as part of a controlled trial. Maintenance sclerotherapy (irrespective of age) consisted of injections every three weeks until all varices were thrombosed. Patients then attended for endoscopy every three months for a year and every six months thereafter. If a patent varix was detected sibsequently follow up every three weeks was reinstituted until all varices were thrombosed. Patients who rebled were managed in the same way as for their first bleed.

\section{Results}

Of the 61 patients aged over 65 at their first presentation, just over half were women. By contrast, among the younger patients there were more men. There was no significant difference in sex ratio with the various aetiologies according to age. Of the over $65 \mathrm{~s}$, $25(41 \%)$ were aged $66-70,21(34 \%)$ were aged $71-75$, $10(16 \%)$ were aged $76-80$, and $5(8 \%)$ were over 80 . Table I shows the sex ratios, aetiologies, and Child's grades of liver disease in the two groups.

Mortality due to the first bleed was higher in younger patients $(39 / 146 ; 27 \%)$ than in older patients $(10 / 61 ; 16 \%)$, but this difference was not significant when corrected for Child's grade. During median follow ups of 25 and 15 months in younger and older patients, respectively (range 1-60 months for both groups), crude survival was considerably worse in older patients (figure). Survival corrected for age by life table analysis, however, was virtually identical for both groups, being $65 \%$ at one year and $60 \%$ at two years (figure). In patients over 65 cumulative survival appeared to be better in women than men, being $60 \%$ $v 50 \%$ at one year and $56 \%$ v $38 \%$ at 18 months

TABLE I-Details of patients studied. Figures are numbers (percentages) of patients

\begin{tabular}{lcc}
\hline & \multicolumn{2}{c}{ Age group (years) } \\
\cline { 2 - 3 } & $\leqslant 65$ & $>65$ \\
\hline No of patients & 146 & 61 \\
Men:women & $85: 61$ & $28: 33$ \\
Aetiology of liver disease: & $68(47)$ & $12(20)$ \\
$\quad$ Alcohol & $19(13)$ & $21(34)$ \\
Cryptogenic & $22(15)$ & $10(16)$ \\
Primary biliary cirrhosis & $12(8)$ & $5(8)$ \\
Chronic active hepatitis & $15(10)$ & $5(8)$ \\
Extrahepatic & $10(7)$ & $8(13)$ \\
Miscellaneous & & \\
Child's grade: & $49(34)$ & $19(31)$ \\
A & $46(32)$ & $28(46)$ \\
B & $51(35)$ & $14(23)$ \\
C & & \\
\hline
\end{tabular}
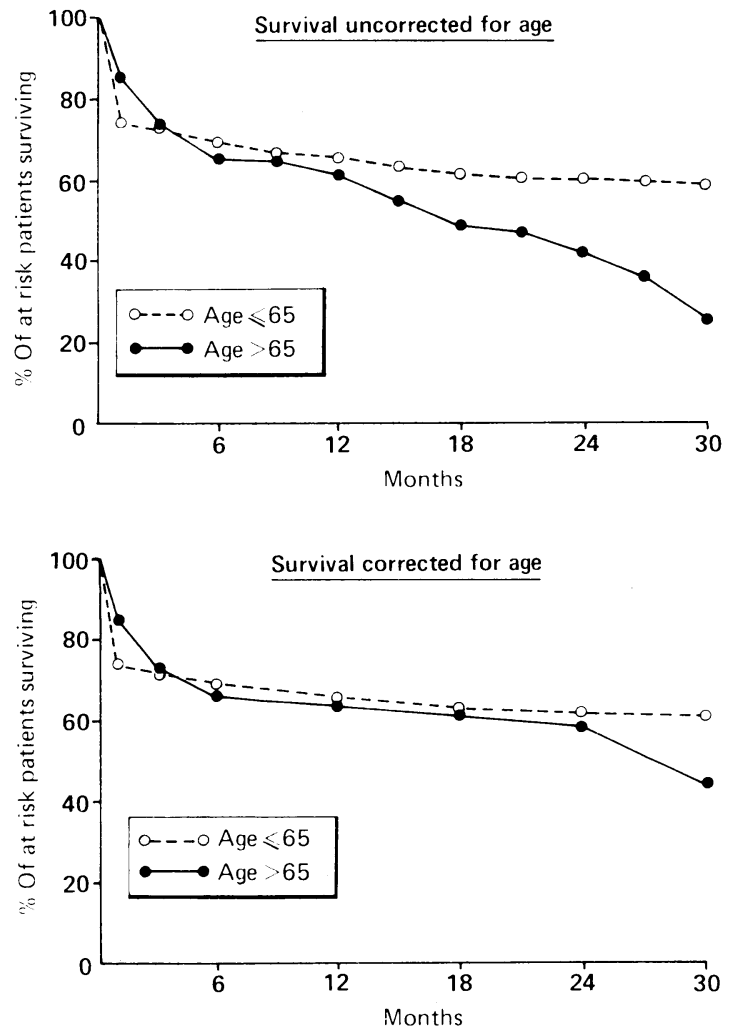

Crude cumulative survival (top) and age corrected (actuarial) survival in young and old patients 
respectively, though these differences may be slightly misleading owing to the greater natural longevity of women. During the whole period 40 of 66 deaths in patients aged under 65 were related to bleeding compared with only 12 of the 38 deaths in the older group ( $\psi^{2}$ test, $\mathrm{p}<0.001$ ); in these intercurrent disease was the more common cause. Analysis of patients aged over 70 compared with those aged 66-70 showed a similar outcome, except that deaths related to bleeding accounted for one fifth of all deaths in the older group. The equivalent figure in the group aged $66-70$ was $52 \%$ (13 deaths). Virtually all deaths related to bleeding occurred within six months of initial presentation. Rebleeding occurred in a greater proportion of elderly patients $\left(\%^{2}, \mathrm{p}<0.01\right)$ and also more often (table II).

TABI.E II - Treatment and outcome in patients studied. Figures are numbers (percentages) of patients

\begin{tabular}{lcc}
\hline & \multicolumn{3}{c}{ Age group (years) } \\
\cline { 2 - 3 } & $\leqslant 65$ & $>65$ \\
\hline No of patients & 146 & 61 \\
Mortality due to first bleed & $39(27)$ & $10(16)$ \\
Patients requiring balloon tamponade & $51(35)$ & $16(26)$ \\
Emergency surgery for bleeding & $53)$ & $4(7)$ \\
Patients who rebled & $6142)$ & $38(62)$ \\
Bleeding risk factor per patient month & $0 \cdot 09$ & $0 \cdot 12$ \\
Deaths (bleeding related: non-bleeding-related $)$ & $36: 8$ & $10: 10$ \\
Months 0-6 & $1: 4$ & $1: 4$ \\
Months 7-12 & $1: 3$ & $1: 3$ \\
Months 13-18 & $1: 0$ & $0: 2$ \\
Months 19-24 & & \\
\hline
\end{tabular}

\section{Comment}

These results show that elderly patients with bleeding varices managed in an active sclerotherapy programme have a similar outcome to younger patients managed the same way. Twenty four $(40 \%)$ of the elderly patients were transferred from other hospitals, but as survival in these was similar to patients from our own hospital, bias within groups seems unlikely. Though the aetiology of liver disease was in different proportions in the two groups, analysis of survival within subgroups showed it to be independent of aetiology. Based on the evidence of clinical trials, most younger patients with bleeding varices are now managed-at least initially-by injection sclerotherapy. ${ }^{23}$ Not unreasonably, clinicians caring for the elderly have been reserved about the benefits of sclerotherapy in their patients, as these trials included very few elderly patients. Also such patients are often admitted to geriatric wards where emergency access to endoscopy is not readily available; and endoscopy is an essential requirement for sclerotherapy.

Our results showing the benefits of active management of bleeding varices in the elderly are supported by a study in Cologne of 61 elderly patients. ${ }^{+}$Though full details of recruitment of patients were not given, those workers achieved $50 \%$ survival in both young and old groups at 24 months using a similar regimen to ours. The increased rebleeding rate seen in our elderly patients was also noted in a study of 14 patients reported by Roberts et al and led to more hospital admissions but seldom caused death. We emphasise that both the group in Cologne and we performed endoscopy and sclerotherapy within 12 hours of admission to hospital. This not only reduces blood loss but also reduces hepatic decompensation, which is an important cause of death. We believe that active treatment of an elderly patient bleeding from varices is well worth while and should not be denied on the basis of age alone.

1 Hyams DE, Fox RA. The gastrointestinal system-the liver and biliary system. In: Brocklehurst JC, ed. Textbook of geriatric medicine and gerontology Edinburgh: Churchill Livingstone, 1985:557-88.

. Macdougall BRD, Westaby D, Theodossi A, Dawson JL, Williams R Increased long term survival in variceal haemorrhage using injection sclerotheraps. Lancet $1982 ; \mathrm{i}: 124-7$

3 Terblanche J, Bornman PC, Kahn D, et al. Failure of repeated injection sclerotherapy to improve long term survival after oesophageal variceal hlecding 1 inct 1985 .ii:1328-32.

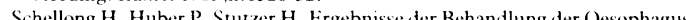
varizenblütung 70)-bis 90) jähriger Patienten: eine prospektive Untersuchung. varizentutung 70 -bis 90 jahriger Paticn

5 Roberts CM. Carev B. Faizallah R, et al. Injection sclerotherapy for oesophageal Roberts CM, Cares B. Faizallah R, et al. Injection scter
varices in the elderly. Age Ageing 1983;12:139-43.

Accepled 7 Norember 1988
Lothian Area Colposcopy Clinic, Elsie Inglis Maternity Hospital, Edinburgh EH8 8HT

M I Alloub, MRCOG, Smith and Nephew research fellow G E Smart, FRCOG, consultant gynaecologist

\section{Departments of} Bacteriology, Pathology, and Dermatology, University of Edinburgh, Edinburgh

B B B Barr, PHD, research assistant

K M McLaren, MRCPATH, senior lecturer

I W Smith, PHD, senior lecturer

M H Bunney, FRCP, research fellow

Correspondence to: $\mathrm{Mr} \mathrm{M} \mathrm{I}$ Alloub, Department of Obstetrics and Gynaecology, Princess Anne Hospital, Southampton.

\section{Human papillomavirus infection and cervical intraepithelial neoplasia in women with renal allografts}

\author{
M I Alloub, B B B Barr, K M McLaren, I W Smith, M H Bunney, G E Smart
}

$16 / 18 \mathrm{DNA}(27 \%$ in the women with allografts and $6 \%$ in the controls).

These data confirm that pathological and virological changes affecting the cervix are significantly increased in immunosuppressed women and emphasise the need for regular colposcopic examination.

\section{Introduction}

An increased prevalence of malignancy in general' and of both intraepithelial and invasive neoplasia of the female genital tract has been observed in immunosuppressed patients. ${ }^{2-5}$ Porreco et al reported a 14-fold increase in the prevalence of cervical intraepithelial neoplasia in women with renal allografts over that in women of the same age in the general population, ${ }^{6}$ and this was confirmed by others." suggested that human papillomavirus might be implicated in the pathogenesis of neoplasia of the lower genital tract." Analysis of the results of a large number 